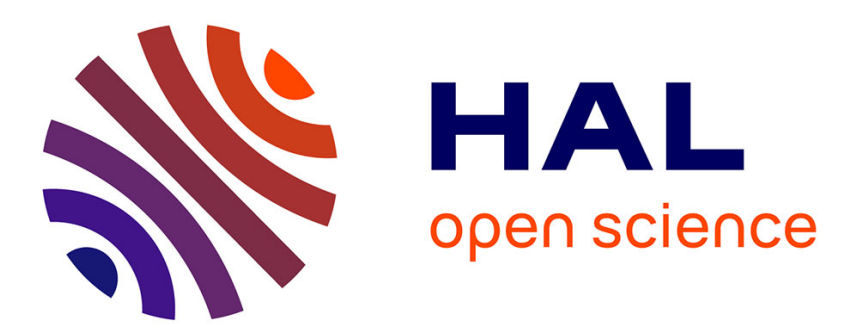

\title{
Leishmania donovani RAN-GTPase interacts at the nuclear rim with linker histone $\mathrm{H} 1$
}

Despina Smirlis, Haralabia Boleti, Maria Gaitanou, Manuel Soto, Ketty

Soteriadou

\section{- To cite this version:}

Despina Smirlis, Haralabia Boleti, Maria Gaitanou, Manuel Soto, Ketty Soteriadou. Leishmania donovani RAN-GTPase interacts at the nuclear rim with linker histone H1. Biochemical Journal, 2009, 424 (3), pp.367-374. 10.1042/BJ20090576 . hal-00479187

\section{HAL Id: hal-00479187 \\ https://hal.science/hal-00479187}

Submitted on 30 Apr 2010

HAL is a multi-disciplinary open access archive for the deposit and dissemination of scientific research documents, whether they are published or not. The documents may come from teaching and research institutions in France or abroad, or from public or private research centers.
L'archive ouverte pluridisciplinaire HAL, est destinée au dépôt et à la diffusion de documents scientifiques de niveau recherche, publiés ou non, émanant des établissements d'enseignement et de recherche français ou étrangers, des laboratoires publics ou privés. 
1

2

3

4

5

6

7

8

9

10

11

12

13

14

15

16

17

18

19

20

21

22

23

24

25

26

27

28

29

30

31

32

33

34

35

36

37

38

39

40

41

42

43

\section{LEISHMANIA DONOVANI RAN-GTPase INTERACTS AT THE NUCLEAR} RIM WITH LINKER HISTONE H1

Despina Smirlis*, Haralabia Boleti $* \S$, Maria Gaitanou $\uparrow$, Manuel Soto $\ddagger$, Ketty Soteriadou*

*Laboratory of Molecular Parasitology, Department of Microbiology, $†$ Laboratory of Cellular and Molecular Neurobiology, Department of Biochemistry, ${ }^{\S}$ Light Microscopy Unit, Hellenic Pasteur Institute, 127 Bas. Sofias Ave., 11521, Athens, Greece; + Centro de Biología Molecular Severo Ochoa (CSIC-UAM), Departamento de Biología Molecular, Universidad Autónoma de Madrid, 28049 Madrid, Spain

Address correspondence to: Despina Smirlis, Laboratory of Molecular Parasitology, Department of Microbiology, Hellenic Pasteur Institute, 127 Bas. Sofias Ave., 11521, Athens, Greece, Tel: +302106478879 Fax: + 30210 6426323; e-mail address: penny@pasteur.gr 


\section{SYNOPSIS}

Ran-GTPase regulates multiple cellular processes such as nucleo-cytoplasmic transport, mitotic spindle assembly, nuclear envelope assembly, cell-cycle progression and the mitotic checkpoint. The leishmanial Ran protein contrary to its mammalian counterpart which is predominately nucleoplasmic is localised at the nuclear rim. The focus of this paper was to characterise the L.donovani Ran orthologue ( $L d \mathrm{Ran}$ ) with emphasis on the Ran-histone association. $L d$ Ran was found to be developmentally regulated, expressed three times less in the amastigote stage. $L d$ Ran over-expression caused a growth defect linked to a delayed Sphase progression in promastigotes like its mammalian counterpart. We report for the first time that Ran interacts with a linker histone -histone H1- in vitro and that the two proteins co-localise at the parasite nuclear rim. Interaction of Ran with core histones $\mathrm{H} 3$ and H4, creating in metazoans a chromosomal Ran-GTP gradient important for mitotic spindle assembly, is speculative in Leishmania spp., not only because this parasite undergoes a closed mitosis but also because the main localisation of $L d$ Ran is different from that of core histone H3. Interaction of Ran with the leishmanial linker histone H1 (LeishH1), suggests that this association maybe involved in modulation of other pathways than those documented for the metazoan Ran-core histone association.

\section{KEYWORDS}

Leishmania, cell-cycle, chromosomal gradient, over-expression.

\section{SHORT TITLE}

$L d$ Ran at the nuclear rim interacts with histone $\mathrm{H} 1$

\section{ABBREVIATIONS FOOTNOTE}

$\mathrm{Ab}$, antibody; pAb, polyclonal antibody; mAb, monoclonal antibody; CAS, Cellular Apoptosis Susceptibility; GFP, green fluorescent protein; GST, glutathione transferase; NTF2, Nuclear Factor Factor 2; Ran, RAs-related Nuclear protein; Ran binding protein 1, RanBP1; RCC1, Regulator of chromosome condensation 1.

\section{INTRODUCTION}

Ran-GTPase belongs to the Ras superfamily of monomeric $G$ proteins that switches between a GDP- and a GTP- bound form [1]. The transition from Ran-GDP to Ran-GTP occurs only by nucleotide exchange. The nuclear exchange factor RCC1 catalyses this reaction and results in efficient generation of nuclear Ran-GTP [2]. The conversion of Ran-GTP to RanGDP is catalyzed in the cytosol by Ran-GAP1, which activates Ran's intrinsic GTPase activity [3]. Ran is involved in multiple cellular processes such as modulation of nucleocytoplasmic transport of macromolecules across the nuclear envelope [4], mitotic spindle assembly [5], post-mitotic nuclear envelope assembly [6], cell-cycle progression [7] and the mitotic checkpoint [8].

The predominant localisation of Ran-GTPase in most eukaryotic cells is in the nucleoplasm, where it is mostly found in the GTP bound form [7]. The Ran-GTP gradient across the interphase nuclear envelope and on the condensed mitotic chromosomes is essential for many cellular processes, including nucleo-cytoplasmic transport and spindle assembly [9]. The mammalian Ran-GTPase is known to interact in the nucleoplasm with chromatin. This interaction occurs via two distinct mechanisms. One mechanism is the interaction of Ran with its nucleotide exchange factor RCC1 which in turn interacts with histones $\mathrm{H} 2 \mathrm{~A}$ and $\mathrm{H} 2 \mathrm{~B}$ [10] and the other via a direct binding of Ran to histone $\mathrm{H} 3$ and histone H4 [11]. The Ran-RCC1 binary complex binds stably to chromatin and ensures that RCC1 couples its guanylyl exchange factor (GEF) activity to chromosome binding [12]. Via 
94 these core histone-Ran/RCC1 interactions, at least in animal cells, Ran-GTP appears to form 95 during mitosis a gradient with the highest concentration on the condensed chromosomes that 96 tapers off towards the periphery of the cell [12]. Experiments in Xenopus egg extracts further suggest that a high Ran-GTP concentration near the chromosomes stimulates microtubule nucleation, whereas microtubule stabilisation is favoured by the lower concentration of RanGTP found farther away from the chromosomes [13]. These differential effects of Ran-GTP on microtubules could be critical for spindle assembly. Taking together these findings indicate that the mitotic Ran-GTP chromosomal concentration gradient is important to navigate spindle assembly towards the condensed, RCC1-rich chromosomes in animal cells.

The RanGTP-chromosomal gradient is not so evident in systems where Ran is not predominantly nucleoplasmic. Only a few examples of non-nucleoplasmic localization of Ran are known to date. One such example is the localisation of the Ran2 protein of Arabidopsis, a plant orthologue of Ran localised in the nuclear envelope/rim and in perinuclear structures [14]. Another example is T. gondii's Ran-GTPase orthologue, that was detected throughout the cell [15]. Additionally the trypanosomatid L. major Ran-GTPase ( $L m j \mathrm{Ran}$ ) fused to GFP was recently found to decorat
closely associated with nuclear pore complexes [16].

Leishmania is a protozoan parasite, a member of the Trypanosomatidae family, which is responsible for a spectrum of diseases in man. Depending on the Leishmania species and on the immunological response of the host, the disease ranges from self-healing skin lesions to life-threatening visceral leishmaniasis causing extensive morbidity and mortality [17]. Fourteen million people are infected with Leishmania with an estimated yearly incidence of 1.5-2 million new cases [17]. Leishmania is transmitted by the blood sucking phlebotomine sand fly. During its life-cycle the parasite exists in two forms, as extracellular flagellated promastigote in the insect vector and in the non-motile amastigote form in the acidic phagolysosome of the macrophage in the mammalian host [18]. Recent advances in parasite differentiation and survival strategies within the macrophages have facilitated the understanding of key aspects in Leishmania pathogenesis, although many more remain unknown (reviewed in 19).

The fundamental processes of cell-biology mediated by Ran-GTPase are expected to play a crucial role in survival and growth strategies of the Trypanosomatid parasites. A Ran orthologue in T. brucei, rtb2 [20], has been shown to be an essential gene for parasite survival [16]. The L. major orthologue was recently identified and was shown to co-localise at the nuclear membrane with the homologue of nucleoporin Sec13 [16]. Several potential partners of $L m j$ Ran have been identified by BLAST search (NTF2, CAS, RANBP1) and their localisation matches the nuclear envelope localisation of $L m j \operatorname{Ran}$ [16].

This paper describes the investigation of a $L d$ Ran interaction with the leishmanial histones H1, H2B and H3 (LeishH1, LeishH2B and LeishH3 respectively). $L d$ Ran was found to specifically interact with LeishH1 and co-localise with this histone at the nuclear rim. This is the first evidence of an interaction of a Ran protein with a linker histone opening the field to a more in depth investigation on the purpose of this interaction to the parasite's cell biology.

\section{EXPERIMENTAL:}

Plasmids

The gene encoding $L d$ Ran (Genebank accession EU456549) was amplified by PCR, from genomic L. donovani (MHOM /ET/ 0000/ HUSSEN) DNA. The forward and reverse primers used were the ${ }^{5}$ TTT TGG AAT TCT ATG CAA CAG GCA CCC TCG ${ }^{3}$ and the ${ }^{5}$ ATG 
GGC GAT GAC GAG GGA CTC GAG GCCG ${ }^{3}$ ' respectively, based on the L. infantum

145

146

147

148

149

150

151

152

153

154

155

156

157

158

159

160

161

162

163

164

165

166

167

168

169

170

171

172

173

174

175

176

177

178

179

180

181

182

183

184

185

186

187

188

189

190

191

192

DNA sequence. The PCR product was cloned in the EcoRI and XhoI site of the pTriex1.1 (Novagen, Merck KGaA, Darmstadt, Germany), in frame with the C-terminal -6 His tag to generate the pTriex- $L d$ Ran plasmid.

For the generation of a leishmanial $L d$ Ran expression plasmid, the $L d$ Ran encoding DNA was amplified from genomic L. donovani (MHOM/ET/0000/HUSSEN) DNA by using as forward and reverse primers the ${ }^{5}$ GCA CGG ATC CGT ACA CCA TGC AAC AGG CAC $\mathrm{C}^{3^{\prime}}$ and the ${ }^{5^{\prime}}$ GAC ACT CGA GGG GTC TCA CTC GTC ATC ${ }^{3^{\prime}}$ respectively. The PCR product was then digested with BamHI and XhoI and inserted in the BglII and XhoI site of the LEXSY-SAT vector, to generate the $L d$ Ran-SAT plasmid.

Murine Rabla (accession number AF226873) cDNA was amplified by RT-PCR using the forward and reverse primers, ${ }^{5^{\prime}}$ CGC GGA TCC ATG TCC AGC ATG AAT CCC G ${ }^{3^{\prime}}$ and ${ }^{5}$ ATA AGA ATG CGG CCG CTT AGC AGC AGC C ${ }^{3^{\prime}}$ respectively. The amplified product was cloned in the BamHI and NotI restriction sites of pGEX4T1 plasmid as a fusion protein with Glutathione-S-Transferase (GST).

The Leishmania Histone H1 (LeishH1) gene was cloned in the pGEX-4T1 as previously described [21]. The myo-inositol-1-phosphate synthase gene (INO1) was cloned in the pTriex1.1 palsmid as previously described [22].

\section{Cell Culture and transfection}

L. donovani (MHOM/ET/0000/HUSSEN) promastigotes were cultured in Medium 199 containing $10 \% \mathrm{v} / \mathrm{v}$ heat inactivated foetal bovine serum (HIFBS) at $26^{\circ} \mathrm{C}$ as previously described [21]. L. donovani parasites were transfected with the Leishmania expression plasmids (supercoiled, transfected as episomes) SAT, $L d$ Ran-SAT as previously described [21]. For selection of transgenic parasites $100 \mu \mathrm{g} / \mathrm{ml}$ of noursethricin (Jena Bioscience, Jena, Germany) was used. To assess the growth of these parasites, parasites were immobilised by addition of $30 \mu 13.7 \%$ formaldehyde in $1 \mathrm{ml}$ phosphate buffered saline (PBS), and counted in a malassez haemocytometer.

Axenic L. donovani amastigotes were generated as previously described [21].

\section{SDS-polyacrylamide gel electrophoresis and immunoblotting}

SDS-polyacrylamide gel electrophoresis (SDS-PAGE) was performed by the method of Laemmli [23]. For immunonoblotting proteins were transferred on a nitrocellulose filter (Hybond C, Amersham Biosciences) and immunoblotting was performed as previously described with the use of 3,3 diaminobenzidine as a chromometric substrate [24] or by enhanced chemiluminescence (ECL Plus, GE Healthcare) according to the manufacturers instructions. For the quantification of immunoblot bands, the Alpha imager software (Alpha Innotech) was used.

\section{Production of recombinant proteins and generation of antibodies}

Recombinant $L d$ Ran and LinINO1 were generated as C- terminal -6 His tagged proteins in the Escherichia coli strain BL21pLysS, as previously described [21] and the recombinant proteins were purified on a Ni-NTA matrix under denaturing conditions according to the manufacturer's instructions (Qiagen, Valencia, CA, USA).

$300 \mu \mathrm{g}$ (LdRan and INO1) and $30 \mu \mathrm{g}$ [(LeishH2B, LeishH3),[25]] and LdRan] of recombinant proteins were used per injection for the immunisation of 2 New Zealand white rabbits and $2 \mathrm{Balb} / \mathrm{c}$ mice respectively for each protein, as previously described [21]. Affinity purified Abs (anti-LdRan, anti-LeishH1, anti-LeishH2B and anti-LeishH3) were isolated by low $\mathrm{pH}$ elution from nitrocellulose strips with purified $L d \mathrm{Ran}$, LeishH1, LeishH2B and 
193 LeishH3 respectively, as previously described [21]. A second step of affinity purification of

194

195

196

197

198

199

200

201

202

203

204

205

206

207

208

209

210

211

212

213

214

215

216

217

218

219

220

221

222

223

224

225

226

227

228

229

230

231

232

233

234

235

236

237

238

239

240

241

242

the anti- $L d \mathrm{R}$ an $\mathrm{Ab}$, was followed to ensure its specificity.

\section{Immunofluorescence}

L. donovani promastigotes $\left(3-5 * 10^{6} / \mathrm{ml}\right)$ were washed once with PBS and then fixed $(20 \mathrm{~min}$, RT) with PBS containing $2 \%(\mathrm{w} / \mathrm{v})$ paraformaldehyde (PF) or with ice cold methanol for 5 min. The parasite cells were then permeabilised and blocked by incubation $(1 \mathrm{~h}$, room temperature $)$ with blocking buffer $(0.3 \% \mathrm{w} / \mathrm{v}$ Bovine serum albumin, $0.1 \%$ Triton-X-100 in PBS) and were subsequently stained with the affinity purified anti- $L d \operatorname{Ran} A b(0.2 \mu \mathrm{g} / \mathrm{ml})$ diluted in blocking buffer. For co-localisation studies affinity purified rabbit anti-LeishH1 [21], mouse anti-LeishH2B, anti-LeishH3 and anti- $L d$ Ran pAbs were used at concentrations ranging from $2-10 \mu \mathrm{g} / \mathrm{ml}$. The commercially available $\mathrm{mAb}$ specific for nuclear pore complex proteins that recognises the conserved FXFG repeats in nucleoporins (Abcam, Cambridge, MA, USA) was used at a final concentration of $10 \mu \mathrm{g} / \mathrm{ml}$. Incubation with the primary Abs, was performed overnight in a humid chamber at $4^{\circ} \mathrm{C}$. After extensive washing the appropriate secondary Abs were added, anti-rabbit and anti-mouse Alexa 546 and Alexa 488 (Molecular Probes, Invitrogen, Carlsbad, CA, USA) at a final concentration of $2 \mu \mathrm{g} / \mathrm{ml}$ blocking buffer, for $2 \mathrm{hs}$ at room temperature. The secondary $\mathrm{Ab}$ was removed with extensive washing and the parasite DNA was stained (10 min, at room temperature) with $10 \mu \mathrm{g} / \mathrm{ml}$ propidium iodide (PI) solution in PBS containing $100 \mu \mathrm{g} / \mathrm{ml}$ RNAse. Samples were washed twice with PBS and the coverslips were mounted with Mowiol. Microscopic analysis of the samples was performed by a Leica TCS SP confocal microscope using the $63 \mathrm{X}$ apochromat lens.

For quantifying co-localisation of LdRan with LeishH1 the Pearson's correlation coefficient (r) and the Red/Green and Green/Red pixel correlation, were calculated by the Intensity Correlation Analysis program ImagePro 5 software (Media Cybernetics) from a typical image out of at least 15 cells from 3 independently performed experiments.

\section{Cell-synchronisation and flow cytometry}

L. donovani parasites in the logarithmic phase $\left(\sim 5 * 10^{6} / \mathrm{ml}\right)$ were synchronised with $5 \mathrm{mM}$ hydroxyurea (HU) in the G1/S border of the cell-cycle, as previously described [21]. PI labelling and Flow Cytometry (FACS) analysis in a FACS Calibur Flow Cytometer (BectonDickinson Immunocytometer System, San Jose, CA, USA) were performed as previously described [21].

\section{LdRan and GST-LeishH1 pull-down assays}

$L d$ Ran was purified under denaturing conditions (6M urea), as described above, and maintained bound to the Ni-NTA beads ( $\sim 2 \mu \mathrm{g}$ of $L d$ Ran per reaction). The urea was removed by washing the beads 5 times with 10 bed volumes of PBS pH 8 . The beads were finally resuspended in $1 \mathrm{ml}$ leishmanial protein extract $(2 \mathrm{mg} / \mathrm{ml})$ in PBS $\mathrm{pH} 8$, containing $5 \mathrm{mM}$ $\mathrm{MgCl}_{2}, 1 \mathrm{mM}$ PMSF, $2.5 \mu \mathrm{g} / \mathrm{ml}$ aprotinin and $1 \mu \mathrm{g} / \mathrm{ml}$ pepstatin. As a control, $2 \mu \mathrm{g}$ of GST or GST-Rabla were immobilised on glutathione sepharose 4B beads (Amersham) and incubated with $1 \mathrm{ml}$ parasite protein extract $(2 \mathrm{mg} / \mathrm{ml})$. The binding reaction was performed at room temperature for 3 hs. Subsequently, unbound proteins were removed by centrifugation and the beads were washed four times with 10 volumes of PBS $\mathrm{pH} 8$. Finally, proteins bound to the beads, were eluted with an equal volume of elution buffer $\left(50 \mathrm{mM} \mathrm{Na}_{2} \mathrm{PO}_{4} \mathrm{pH} 8,300 \mathrm{mM}\right.$ $\mathrm{NaCl}, 250 \mathrm{mM}$ imidazole) three times.

For the GST-LeishH1 pull-down assays, $2 \mu \mathrm{g}$ of GST and GST-LeishH1 [21] were immobilised on $50 \mu \mathrm{l}$ of glutathione sepharose 4B beads according to the manufacturer's instruction (Amersham Pharmacia Biochem, St Albans, Herts, UK) and incubated for 3 hs at room temperature with $1 \mathrm{ml}$ leishmanial protein extract $(2 \mathrm{mg} / \mathrm{ml})$ in PBS containing $5 \mathrm{mM}$ 
243

244

245

246

247

248

249

250

251

252

253

254

255

256

257

258

259

260

261

262

263

264

265

266

267

268

269

270

271

272

273

274

275

276

277

278

279

280

281

282

283

284

285

286

287

288

289

290

291

292

$\mathrm{MgCl}_{2}, 1 \%(\mathrm{v} / \mathrm{v})$ Triton-X-100, $1 \mathrm{mM}$ PMSF, $2.5 \mu \mathrm{g} / \mathrm{ml}$ aprotinin and $1 \mu \mathrm{g} / \mathrm{ml}$ pepstatin. Glutathione sepharose beads were subsequently washed 4 times with 20 volumes of PBS containing $5 \mathrm{mM} \mathrm{MgCl} 2$ and $1 \%(\mathrm{v} / \mathrm{v})$ Triton-X-100 and then frozen at $-20^{\circ} \mathrm{C}$ prior to their analysis by SDS/PAGE and Western Blot.

\section{RESULTS:}

Identification and characterisation of LdRan; LdRan is developmentally regulated An open reading frame (ORF) encoding a putative leishmanial Ran orthologue was identified (LinJ25.1470) as a single locus on chromosome 25, in the Leishmania infantum (L. infantum) genome after a search in the Leishmania GeneDB database. Primers based on the L. infantum ORF LinJ25.1470, were designed and the putative L. donovani Ran (LdRan) gene was amplified and cloned in the bacterial expression plasmid pTriex with a 6 -His C-terminal tag.

The putative $L d$ Ran protein (GenBank accession number EU426549), was identical to the L. infantum and L. major Ran (LinRan and LmjRan respectively) orthologues. Amino acid sequence alignment of $L d$ Ran with Ran proteins from different species showed that it is highly conserved, having an $80 \%$ aminoacid sequence identity with Ran orthologues from species as distant as Homo sapiens. Western blot analysis using the generated anti-LdRan specific $\mathrm{Ab}$ showed that $L d$ Ran is expressed in $L$. donovani promastigotes as a $\sim 25 \mathrm{kDa}$ protein, in agreement with the predicted molecular weight $(24,223 \mathrm{kDa})$ (fig.1A).

$L d$ Ran expression was also evaluated in axenic amastigotes [21] by western blot analysis using the $L d$ Ran specific Ab (Fig. 1A) and by immunofluorescence (data not shown). Scanning densitometry of the detected bands revealed that $L d$ Ran expression level was 3-fold lower in amastigotes. As a control for loading equal number of cells, the blot was probed with the antiserum against $L$. infantum myo-inositol-1-phosphate synthase (LinINO1), a $46 \mathrm{kDa}$ protein which is equally expressed in promastigotes and amastigotes [26] (Fig.1A1). Expression of A2 proteins was also checked with the anti-A2 C9 mAb to ensure that axenic amastigotes had properly differentiated and expressed amastigote specific proteins as expected (fig.1A2).

\section{Over-expression of LdRan delays cell-cycle progression in Leishmania}

$L d$ Ran was over-expressed by stable transfection of $L$. donovani parasites with an episomal plasmid. Parasites were viable with no apparent morphological differences from control parasites (bearing plasmid alone, SAT). Over-expression of $L d$ Ran, in the $L d$ Ran-SAT parasites as compared to control parasites, was assessed by Western Blot analysis and quantification by densitometry which showed a three fold (Fig.1B) over-expression. Equal loading was confirmed with the use of an anti-INO1 Ab as a loading control (Fig.1B).

The growth curve of these promastigotes indicated a mild but consistent delay in the logarithmic phase of their growth (Supplementary Fig. 1A), suggesting that $L d$ Ran overexpression delays cell division. This effect was more pronounced upon host-free differentiation conditions (Supplementary Fig. 1B). Annexin V-PI staining showed that there was no significant difference in early apoptotic and necrotic (or late apoptotic) control and $L d$ Ran over-expressing parasites (data not shown). This confirms that the effect is solely due to a delay in growth and not due to increased cell death.

To determine which phase of the cell-cycle is affected by $L d$ Ran over-expression we evaluated by flow cytometry, the cell-cycle progression of $L$. donovani promastigotes overexpressing $L d$ Ran ( $L d$ Ran-SAT) and compared it to cell-cycle profiles of control parasites (SAT). SAT and $L d$ Ran-SAT $L$. donovani logarithmic parasites were synchronised in the G1/S phase of the cell-cycle with hydroxyurea (HU, Fig.2 and Table 1). Both SAT and $L d$ Ran-SAT HU synchronised parasites had a greater percentage of cells in the G0/G1 phase 
293 of cell-cycle ( $72 \%$ and $71 \%$ respectively) as compared to the G0/G1 phase of the same

294

295

296

297

298

299

300

301

302

303

304

305

306

307

308

309

310

311

312

313

314

315

316

317

318

319

320

321

322

323

324

325

326

327

328

329

330

331

332

333

334

335

336

337

338

339

340

341

342 asynchronous logarithmic parasites (55\% and 52\% respectively), as expected. Four hours post-release more parasites over-expressing $L d$ Ran were in the $\mathrm{G} 1 / \mathrm{S}$ border $(35 \%)$ as compared to control parasites (27\%, fig. 2 , table 1$)$. Six hours post-release $31 \%$ and $50 \%$ of control parasites were in the S and G2/M phases of the cell-cycle whereas $41 \%$ and $33 \%$ of $L d$ Ran over-expressing parasites were in the $\mathrm{S}$ and G2/M phases respectively (Fig.2, Table 1). Therefore, $L d$ Ran over- expressing parasites show a delay in the completion of $S$ phase. Ten hours post-release there was still a greater percentage of $L d$ Ran over-expressing parasites in the S phase, as compared to control (30\% versus $18 \%$ respectively), confirming that $L d$ Ran over-expression causes a constant deregulation of $\mathrm{S}$ phase progression (confirmed in all experiments). This delay in the phases of the cell-cycle was calculated to be approximately 2 hs with respect to the control parasites, a duration that is significant at the promastigote stage, where the parasite completes one cell-cycle between 8-10 hs.

\section{LdRan at the nuclear envelope co-localises with linker histone H1}

The localisation of endogenous Ran in wild-type $L$. donovani promastigotes was assessed using an affinity purified anti- $L d$ Ran primary Ab. Double staining with PI (fig.3A1) or detection of FG nucleoporins (fig.3A2) showed that $L d$ Ran is localised at the nuclear envelope/rim, as it is the case for $L m j \operatorname{Ran}$ [16] which as mentioned above is identical to $L d$ Ran. Quantititative analysis showed using the Image Pro software showed that $95 \%$ of $L d$ Ran co-localises with FG nucleoporins, whereas $70 \%$ of FG nucleoporins co-localises with $L d$ Ran (Fig. 3A2). Expression of $L m j$ Ran as a fusion protein with GFP [16], cloned in the plasmid $\mathrm{pTH}_{6} \mathrm{cGFPn}$ vector [27], in L. donovani showed that that GFP-LmjRan is localized at the vicinity of the nuclear envelope (data not shown) confirming thereby the specificity of the generated anti- $L d$ Ran $A b$ used in immunostaining.

Since $L d$ Ran does not predominantly localise in the nucleoplasm of promastigotes we investigated whether $L d$ Ran associates with histones. First we examined the degree of core histone co-localisation with LaRan. For this study we used histone H3, being one of Ran's binding proteins in mammalian cells. We also investigated $L d$ Ran's co-localisation with LeishH1, knowing from previous studies that this histone had a nuclear rim localisation at least in the majority of parasites (unpublished observation). For this purpose a rabbit anti$L d$ Ran and a mouse anti-LeishH 3 or a rabbit anti-LeishH1 pAb and a mouse anti- $L d$ Ran pAb were used in double immunofluorescence staining experiments (Fig. 3B1 and 3B2 respectively). Figure 3B1 shows that LeishH3 is predominantly localised in the nucleoplasm of the parasite (in $70 \%$ of the cells LeishH3 was nucleoplasmic, and in $30 \%$ of the cells was closer to the nuclear rim), whereas nuclear $L d$ Ran although present in lower concentrations in the nucleoplasm, was predominantly found at the nuclear rim. $L d$ Ran and LeishH3 showed a moderate co-localisation. Quantitative analysis using the Image Pro software showed that $40 \%$ of $L d$ Ran co-localised with LeishH3 and $60 \%$ of LeishH3 co-localised with $L d$ Ran. The Pearson correlation co-efficient indicating the strength and direction of a linear relationship between two random variables, was also moderate $(\mathrm{r}=0.65)$. On the other hand, Fig. 3B3 shows that LeishH1 is localised near the nuclear periphery, and close to the nuclear envelope. This localisation of LeishH1 was not uniform, but was detected in the majority of cells. In more detail, in $75 \%$ of parasites LeishH1 localised at the nuclear rim, where the linker histone did not co-localise with the bulk DNA, and in $25 \%$ of the parasites LeishH1 was nucleoplasmic. LdRan co-localises at the nuclear rim, with LeishH1 (Fig. 3B2). More specifically, quantitative analysis showed that $90 \%$ of LeishH1 co-localised with $L d$ Ran and $80 \%$ of $L d$ Ran co-localised with LeishH1, having a Pearson correlation coefficient of 0.9 . The co-localisation of $L d$ Ran with LeishH1 is significant taking into account the dynamic nature of Ran and histone H1 proteins [28, 29]. This co-localisation was found to be 
343

344

345

346

347

348

349

350

351

352

353

354

355

356

357

358

359

360

361

362

363

364

365

366

367

368

369

370

371

372

373

374

375

376

377

378

379

380

381

382

383

384

385

386

387

388

389

390

391

392 independent of the fixation method, and clearly shows that linker histone H1 may be a candidate partner of $L d$ Ran.

\section{LdRan interacts in vitro with linker histone LeishH1}

To investigate a possible interaction of $L d$ Ran with linker histone $H 1$, we have performed $L d$ Ran pull-down experiments. We examined in parallel the $L d$ Ran's interaction with LeishH3, expecting that these proteins interact in vitro since the mammalian histone H3 globular domain responsible for binding to Ran [11] is well conserved in Leishmania [30]. We also assessed the binding of LeishH2B to $L d$ Ran as a negative control since the mammalian histone H2B is not known to interact directly with Ran, but via RCC1 [10]. As shown in Fig. 4A $L d$ Ran interacts with LeihH1 and LeishH3, but it does not interact with LeishH2B. The anti-histone Abs detected histones almost equally well in equally loaded protein inputs used for the binding reactions (Fig. 4A). LeishH1 bound equally well to $L d$ Ran as to LeishH3 in vitro. Additionally, to verify that $L d$ Ran interacted directly with linker LeishH1 we have incubated recombinant histone H1 (cleaved with thrombin from the GSTmoiety) with $L d$ Ran bound Ni-NTA beads. LeishH1 was detected on the $L d$ Ran Ni-NTA beads whereas no LeishH1 was immobilised on an equal volume of Ni-NTA beads (data not shown), result supporting the direct interaction between the two proteins.

To examine the specificity of $L d$ Ran interaction with LeishH1, we performed a pulldown assay using murine Rabla, which is $30 \%$ identical and $50 \%$ homologous to $L d$ Ran. Rabla was used as a GST fusion protein and equal amounts of GST, GST-Rabla and $L d$ Ran were immobilised on glutathione sepharose and Ni-NTA beads respectively (Fig. 4B1). LeishH1 was only detected in beads with immobilised $L d$ Ran (Fig. 4B2).

To eliminate the possibility that the in vitro interaction of $L d$ Ran with LeishH1 was due to an "aberrant" refolding of recombinant $L d$ Ran, equal amounts of GST (used as negative control) and GST-LeishH1 were immobilised on glutathione sepharose beads (Fig. 4C) and incubated with leishmanial protein extracts. Additionally equal amounts of GST and GST-LeishH1 that were not incubated with leishmanial extracts were also used as negative control (Fig. 4C). Beads were extensively washed after the completion of the incubation period. Native $L d$ Ran was present only in GST-LeishH1 and not in GST- bound beads (Fig. 4C), indicating that $L d$ Ran interacts specifically with LeishH1. Therefore denaturation and refolding of recombinant $L d$ Ran had no effect on the ability of this protein to interact with LeishH1.

\section{DISCUSSION:}

Focus of this work was to characterise the Ran orthologue from L. donovani, emphasing on its interaction with histones. The three-fold down-regulation of $L d$ Ran in axenic amastigotes was in accordance with results from previous studies showing that the mRNA encoding the leishmanial Ran was down-regulated in amastigotes by a factor of 2.3 as compared to the promastigote stage [31] and that the $L d$ Ran protein expression level decreased during differentiation [32]. In the amastigote stage the parasites undergo a number of changes including morphological ones, deregulation of cell-cycle progression and reduction in the rate of protein synthesis [33]. Therefore, down-regulation of expression in the amastigote stage of a protein involved in essential cellular functions like regulation of cellular-division, cell-cycle progression and nucleo-cytoplasmic traffic is not an unexpected finding. Over-expression of $L d$ Ran significantly affected the division of parasites during differentiation, suggesting that $L d$ Ran downregulation is required for appropriate promastigote to amastigote differentiation. 
$L$. donovani promastigotes tolerated over-expression of $L d$ Ran, but these parasites also had a growth defect, linked with the delay in S-phase progression. In mammalian cells, expression of mutants stabilizing Ran in its GTP bound form [34], or depletion of RCC1 [35] (which results in the enrichment of the GDP bound form of Ran) both cause a delay in cellcycle progression, indicating that any disturbance in the GTP/GDP bound state of Ran may bring de-regulation in $S$ phase progression [36]. Importin- $\beta$ appears to be dispensable for regulating cell cycle progression [37], but more investigations are required for revealing the precise mechanism by which Ran modulates cell-cycle progression.

$L d$ Ran, like $L m j \operatorname{Ran}[16]$ localises at the nuclear rim where it co-localises with FG nucleoporins. Interestingly, $L d$ Ran expressed in mammalian cells (COS7), localises at the nucleoplasm (data not shown) indicating that the nuclear rim localisation of $L d R$ Ran is due to parasite specific interacting proteins. Some of these proteins could be proteins in the Ran network like NTF-2, CAS and present in the leishmanial nuclear envelope [16].

In metazoan cells Ran interacts in the nucleoplasm with histones and this interaction occurs via two distinct mechanisms. One being a direct interaction of Ran with core histones $\mathrm{H} 3$ and $\mathrm{H} 4$ [11], and the other being its indirect interaction with the other two core histones $\mathrm{H} 2 \mathrm{~A}$ and $\mathrm{H} 2 \mathrm{~B}$ via $\mathrm{RCC} 1$ [10]. The nuclear rim predominant localisation of $L d \mathrm{Ran}$ in $L$. donovani promastigotes raised the question on whether an $L d$ Ran-histone association occured. $L d$ Ran as its mammalian counterpart was able to bind to histone H3 but not to histone H2B in vitro. Core histones in Leishmania however, are present predominately in the nucleoplasm in contrast to the $L d$ Ran localisation at the nuclear periphery. Therefore, the moderate co-localisation of $L d$ Ran with LeishH3 makes their interaction in vivo still speculative. In contrast, LeishH1 was present at the nuclear rim in the majority of cells by at least two methods of cell fixation (data not shown). LdRan and LeishH1 directly interacted in vitro and co-localised at the nuclear rim. This is the first evidence up to date of a linker histone interacting with Ran. It is currently not known if this interaction is unique for Leishmania spp., or if it exists in other organisms. It is known that Ran [14] and histone H1 in plants are both present at the nuclear rim, away from the nucleoplasmic histone H3 [38], but their interaction has not been investigated. Plant histone H1 possesses microtubule organizing activity, forming ring shaped complexes with tubulin at atypical microtubule organizing centres (MTOCs) present in the nuclear periphery of plant cells [38, 39]. A possible explanation for the interaction of $L d$ Ran with LeishH1 at the nuclear periphery would be its involvement in the organisation and elongation of MTs adjacent to the leishmanial nuclear envelope [40].

Interaction of Ran with chromatin in metazoans, has an unknown function in interphase cells. In mitotic cells it is postulated that the Ran-histone association, required for the formation of a RanGTP chromosomal gradient, may play an important role during reassembly of the nuclear envelope by increasing the binding of membranes to chromatin surface [11] and for the formation of the mitotic spindle [9]. In Leishmania the nucleus does not break down during mitosis [40], therefore the requirement of a RanGTP-chromosomal gradient for the post-mitotic nuclear envelope assembly is clearly not required. It has been reported that in the closed mitosis of Aspergillus nidulans, the nuclear pores open allowing passive diffusion of proteins [41]. Thus, the Ran-GTP chromosomal gradient, may be essential even in organisms performing a closed mitosis. Therefore, one cannot exclude the possibility that the Ran-LeishH1 interaction in Leishmania is required to keep a form of an atypical chromosomal nuclear rim Ran-GTP gradient in the nuclear rim chromatin. However, the LdRan-linker histone H1 interaction may modulate other pathways than those documented for the metazoan Ran-core histone association. In L.donovani, LeishH1 regulates cell-cycle progression, promastigote to amastigote differentiation and virulence [21]. Interaction of $L d$ Ran with LeishH1 may be important, for the regulation of these processes. 
The Ran-GTPase appears to be a master regulator and coordinator of events that 444 require intimate crosstalk between chromatin and the cytoplasm, for cell-cycle progression 445 and spindle assembly [42]. In Leishmania these events have similarities but also major 446 differences from other eukaryotes like metazoans and yeast. Further investigation is therefore 447 required to elucidate these mechanisms and to define the precise mechanism of $L d$ Ran 448 participation in the cell-cycle of this parasite, and whether an atypical Ran-GTP 449 chromosomal gradient is achieved. Finally, the atypical Ran network in this parasite may be 450 exploited for anti-leishmanial drug development.

\section{ACKNOWLEDGEMENTS}

453 This work was supported by the Hellenic Pasteur Institute. We would like to thank Prof. 454 Matlashewski for donating us the A2 monoclonal antibody and Dr Patrick Bastien for 455 donating us the $\mathrm{pTH}_{6} \mathrm{cGFPn}$-Ran plasmid. Finally we would like to thank Georgia Konidou 456 for her technical assistance and the production of the anti- $L d$ Ran polyclonal antibodies. 
457

458

459

460

461

462

463

464

465

466

467

468

469

470

471

472

473

474

475

476

477

478

479

480

481

482

483

484

485

486

487

488

489

490

491

492

493

494

495

496

497

498

499

500

501

502

503

504

505

506

\section{REFERENCES}

1 Bischoff, F. R. and Ponstingl, H. (1991) Mitotic regulator protein RCC1 is complexed with a nuclear ras-related polypeptide. Proc. Natl. Acad. Sci. U S A. 88, 10830-10834

2 Bischoff, F. R. and Ponstingl, H. (1991) Catalysis of guanine nucleotide exchange on Ran by the mitotic regulator RCC1. Nature. 354, 80-82

3 Bischoff, F. R., Klebe, C., Kretschmer, J., Wittinghofer, A. and Ponsting1, H. (1994) RanGAP1 induces GTPase activity of nuclear Ras-related Ran. Proc. Natl. Acad. Sci. U S A. 91, 2587-2591

4 Moore, M. S. and Blobel, G. (1994) A G protein involved in nucleocytoplasmic transport: the role of Ran. Trends Biochem. Sci. 19, 211-216

5 Heald, R. and Weis, K. (2000) Spindles get the ran around. Trends Cell Biol. 10, 1-4

6 Hetzer, M., Bilbao-Cortes, D., Walther, T. C., Gruss, O. J. and Mattaj, I. W. (2000)

GTP hydrolysis by Ran is required for nuclear envelope assembly. Mol. Cell. 5, 1013-1024

7 Ren, M., Drivas, G., D'Eustachio, P. and Rush, M. G. (1993) Ran/TC4: a small nuclear GTP-binding protein that regulates DNA synthesis. J. Cell. Biol. 120, 313-323

8 Arnaoutov, A. and Dasso, M. (2003) The Ran GTPase regulates kinetochore function. Dev. Cell. 5, 99-111

9 Zheng, Y. (2004) G protein control of microtubule assembly. Annu. Rev. Cell. Dev. Biol. 20, 867-894

10 Nemergut, M. E., Mizzen, C. A., Stukenberg, T., Allis, C. D. and Macara, I. G. (2001) Chromatin docking and exchange activity enhancement of RCC1 by histones H2A and H2B. Science. 292, 1540-1543

11 Bilbao-Cortes, D., Hetzer, M., Langst, G., Becker, P. B. and Mattaj, I. W. (2002) Ran binds to chromatin by two distinct mechanisms. Curr. Biol. 12, 1151-1156

12 Li, H. Y. and Zheng, Y. (2004) Phosphorylation of RCC1 in mitosis is essential for producing a high RanGTP concentration on chromosomes and for spindle assembly in mammalian cells. Genes Dev. 18, 512-527

13 Caudron, M., Bunt, G., Bastiaens, P. and Karsenti, E. (2005) Spatial coordination of spindle assembly by chromosome-mediated signaling gradients. Science. 309, 1373-1376

14 Ma, L., Hong, Z. and Zhang, Z. (2007) Perinuclear and nuclear envelope localizations of Arabidopsis Ran proteins. Plant. Cell Rep. 26, 1373-1382

15 Frankel, M. B. and Knoll, L. J. (2008) Functional analysis of key nuclear trafficking components reveals an atypical Ran network required for parasite pathogenesis. Mol. Microbiol. 70, 410-420

16 Casanova, M., Portales, P., Blaineau, C., Crobu, L., Bastien, P. and Pages, M. (2008) Inhibition of active nuclear transport is an intrinsic trigger of programmed cell death in trypanosomatids. Cell Death Differ. 15, 1910-1920

17 WHO. (2006) Control of leishmaniasis. Geneva, World Health Organization, agenta item 5.1, document EB118/4. Fact sheet No 116

18 Chang, K. P. and Dwyer, D. M. (1978) Leishmania donovani. Hamster macrophage interactions in vitro: cell entry, intracellular survival, and multiplication of amastigotes. J. Exp. Med. 147, 515-530

19 McConville, M. J. and Handman, E. (2007) The molecular basis of Leishmania pathogenesis. Int. J. Parasitol. 37, 1047-1051

20 Field, M. C., Field, H. and Boothroyd, J. C. (1995) A homologue of the nuclear GTPase ran/TC4 from Trypanosoma brucei. Mol. Biochem. Parasitol. 69, 131-134

21 Smirlis, D., Bisti, S. N., Xingi, E., Konidou, G., Thiakaki, M. and Soteriadou, K. P. (2006) Leishmania histone H1 overexpression delays parasite cell-cycle progression, parasite differentiation and reduces Leishmania infectivity in vivo. Mol. Microbiol. 60, 1457-1473 
22 Xingi, E., Smirlis, D., Myrianthopoulos, V., Magiatis, P., Grant, K. M., Meijer, L., Mikros, E., Skaltsounis, A. L. and Soteriadou, K. (2009) 6-Br-5methylindirubin-3'oxime (5Me-6-BIO) targeting the leishmanial glycogen synthase kinase-3 (GSK-3) short form affects cell-cycle progression and induces apoptosis-like death: Exploitation of GSK-3 for treating leishmaniasis. Int. J. Parasitol

23 Laemmli, U. K. (1970) Cleavage of structural proteins during the assembly of the head of bacteriophage T4. Nature. 227, 680-685

24 Papageorgiou, F. T. and Soteriadou, K. P. (2002) Expression of a novel Leishmania gene encoding a histone H1-like protein in Leishmania major modulates parasite infectivity in vitro. Infect. Immun. 70, 6976-6986

25 Iborra, S., Soto, M., Carrion, J., Alonso, C. and Requena, J. M. (2004) Vaccination with a plasmid DNA cocktail encoding the nucleosomal histones of Leishmania confers protection against murine cutaneous leishmaniosis. Vaccine. 22, 3865-3876

26 Ilg, T. (2002) Generation of myo-inositol-auxotrophic Leishmania mexicana mutants by targeted replacement of the myo-inositol-1-phosphate synthase gene. Mol. Biochem. Parasitol. 120, 151-156

27 Dubessay, P., Blaineau, C., Bastien, P., Tasse, L., Van Dijk, J., Crobu, L. and Pages, M. (2006) Cell cycle-dependent expression regulation by the proteasome pathway and characterization of the nuclear targeting signal of a Leishmania major Kin-13 kinesin. Mol. Microbiol. 59, 1162-1174

28 Bustin, M., Catez, F. and Lim, J. H. (2005) The dynamics of histone H1 function in chromatin. Mol. Cell. 17, 617-620

29 Li, H. Y., Wirtz, D. and Zheng, Y. (2003) A mechanism of coupling RCC1 mobility to RanGTP production on the chromatin in vivo. J Cell Biol. 160, 635-644

30 Soto, M., Requena, J. M., Morales, G. and Alonso, C. (1994) The Leishmania infantum histone $\mathrm{H} 3$ possesses an extremely divergent $\mathrm{N}$-terminal domain. Biochim. Biophys. Acta. 1219, 533-535

31 Leifso, K., Cohen-Freue, G., Dogra, N., Murray, A. and McMaster, W. R. (2007) Genomic and proteomic expression analysis of Leishmania promastigote and amastigote life stages: the Leishmania genome is constitutively expressed. Mol. Biochem. Parasitol. 152, 3546

32 Rosenzweig, D., Smith, D., Opperdoes, F., Stern, S., Olafson, R. W. and Zilberstein, D. (2008) Retooling Leishmania metabolism: from sand fly gut to human macrophage. Faseb J. 22, 590-602

33 Gupta, N., Goyal, N. and Rastogi, A. K. (2001) In vitro cultivation and characterization of axenic amastigotes of Leishmania. Trends Parasitol. 17, 150-153

34 Ren, M., Coutavas, E., D'Eustachio, P. and Rush, M. G. (1994) Effects of mutant Ran/TC4 proteins on cell cycle progression. Mol. Cell. Biol. 14, 4216-4224

35 Dasso, M., Nishitani, H., Kornbluth, S., Nishimoto, T. and Newport, J. W. (1992) $\mathrm{RCC} 1$, a regulator of mitosis, is essential for DNA replication. Mol. Cell. Biol. 12, 33373345

36 Moore, J. D. (2001) The Ran-GTPase and cell-cycle control. Bioessays. 23, 77-85

37 Li, H. Y., Cao, K. and Zheng, Y. (2003) Ran in the spindle checkpoint: a new function for a versatile GTPase. Trends Cell. Biol. 13, 553-557

38 Hotta, T., Haraguchi, T. and Mizuno, K. (2007) A novel function of plant histone H1: microtubule nucleation and continuous plus end association. Cell Struct. Funct. 32, 79-87

39 Nakayama, T., Ishii, T., Hotta, T. and Mizuno, K. (2008) Radial microtubule organization by histone $\mathrm{H} 1$ on nuclei of cultured tobacco BY-2 cells. J. Biol. Chem.

40 Triemer, R. E., Fritrz, L. M. and Herman, R. (1986) Ultrastructural features of mitosis in Leishmania Adleri. Protoplasma. 134, 134-162 
55741 De Souza, C. P. and Osmani, S. A. (2007) Mitosis, not just open or closed. Eukaryot. 558 Cell. 6, 1521-1527

55942 Clarke, P. R. and Zhang, C. (2008) Spatial and temporal coordination of mitosis by 560 Ran GTPase. Nat. Rev. Mol. Cell. Biol. 9, 464-477

561 
562

563

564

565

566

567

568

569

570

571

572

573

574

575

576

577

578

579

580

581

582

583

584

585

586

587

588

589

590

591

592

593

594

595

596

597

598

599

600

601

602

603

604

605

606

607

608

609

610

611

Table 1

\section{Cell-cycle distribution after HU withdrawal in $L$. donovani LdRan-SAT and SAT transfectants}

\begin{tabular}{lcccccc}
\hline & \multicolumn{3}{c}{ SAT } & \multicolumn{3}{c}{ LdRan-SAT } \\
\hline & G0/G & S & G2/M & G0/G1 & S & G2/M \\
Asynchronous & $55 \%$ & $12 \%$ & $33 \%$ & $52 \%$ & $12 \%$ & $36 \%$ \\
HU synchronised & $72 \%$ & $11 \%$ & $16 \%$ & $71 \%$ & $9 \%$ & $19 \%$ \\
4h & $27 \%$ & $45 \%$ & $28 \%$ & $35 \%$ & $43 \%$ & $22 \%$ \\
6h & $19 \%$ & $31 \%$ & $50 \%$ & $25 \%$ & $41 \%^{*}$ & $33 \% *$ \\
$\mathbf{1 0 h}$ & $39 \%$ & $18 \%$ & $49 \%$ & $34 \%$ & $30 \% *$ & $35 \% *$
\end{tabular}

Values are from one representative experiment performed 4 times. All 4 experiments showed a consistent $8-12 \%$ difference between the control SAT parasite population and the $L d$ RanSAT population found in the S and G2/M phases 6 and 10 hs post-HU release.* Significantly different from the corresponding control values (SAT), $\mathrm{p}<0.05$ (two tailed, paired Student's ttest). 
612

613

614

615

616

617

618

619

620

621

622

623

624

625

626

627

628

629

630

631

632

633

634

635

636

637

638

639

640

641

642

643

644

645

646

647

648

649

650

651

652

653

654

655

656

657

658

659

660

\section{FIGURE LEGENDS:}

\section{Fig. 1}

Expression of $L d$ Ran in promastigotes and axenic amastigotes and over-expression of $L d$ Ran in $L d$ Ran-SAT transfected $L$. donovani parasites

A. Leishmania extracts from promastigotes (P) and axenic amastigotes (A) were analyzed by SDS-PAGE and subsequently by Western Blot. Total cell extracts from $10^{7}$ promastigotes or amastigotes were loaded per lane. 1) Detection of $L d$ Ran protein expression in promastigotes and axenic amastigotes using the anti-LdRan specific $\mathrm{pAb}$ and an anti-LinINO1 $\mathrm{Ab}$ as a loading control 2) Detection of A2 protein expression in axenic amastigotes using the anti-A2 C9 mAb. B. Immunoblot analysis of $10^{7} \mathrm{~L}$. donovani parasites in the stationary phase transfected with either the $L d$ Ran-SAT expression plasmid or with the control plasmid (SAT). To detect $L d$ Ran, the anti- $L d$ Ran Ab was used ( $L d$ Ran). An anti-LinINO1 Ab was used to confirm that equal amounts of parasite extracts were loaded in both lanes. The experiment was performed at least three times. The intensities of the bands were analyzed by the Alpha Imager Software. The fold-over-expression was calculated by dividing the band intensity of $L d$ Ran with the band intensity of INO1 and comparing this ratio in $L d$ RanSAT transfected parasites over the same ratio in control SAT parasites.

\section{Fig. 2}

Cell-cycle analysis after hydroxyurea withdrawal in LdRan-SAT or SAT L. donovani transfectants synchronised in the G1/S border

The DNA content of control parasites bearing plasmid alone (SAT) or over-expressing $L d$ Ran ( $L d$ Ran-SAT) was analyzed by flow cytometry in cells stained with propidium iodide (PI). The cell-cycle distribution in these cells was calculated by the MOD-FIT software. Parasites synchronised with Hydroxyurea (HU) in the G1/S border are indicated as HU synchronised, $0 \mathrm{~h}$. The time points after release of the HU block are indicated on the left. Not synchronised, logarithmically growing parasites are also indicated at the top (asynchronous). Arrowheads at $6 \mathrm{hs}$ show the proportion of parasites with $4 \mathrm{~N}$ DNA content (G2/M). The percentage of this population is less in parasites over-expressing $L d$ Ran. A representative experiment of four independently performed experiments is presented in this figure.

\section{Fig. 3}

\section{Localisation of $L d$ Ran in $L$. donovani promastigotes with respect to histones LeishH1 and LeishH3}

A. Nuclear rim localisation of $L d$ Ran in $L$. donovani promastigotes. 1. Phase contrast (phase) and fluorescence microscopy images in black and white show nuclear and kinetoplast DNA staining with propidium iodide (PI) and $L d$ Ran staining using a primary anti- $L d$ Ran specific $\mathrm{Ab}$ and a secondary anti-rabbit Alexa $488 \mathrm{Ab}$. A two-fold magnification of the nucleus is also shown at the bottom right corner of each image. Merged images of the red (PI) and green fluorescence are shown on the left. 2. Co-localisation of endogenous $L d$ Ran with nucleoporins. Wild-type L. donovani promastigotes were stained for nucleoporins (NUP), with an anti-nucleoporin specific $\mathrm{mAb}$ and an anti-mouse Alexa 488 secondary $\mathrm{Ab}$, and for $L d \operatorname{Ran}(L d \operatorname{Ran})$ with the anti- $L d$ Ran specific rabbit pAb and an anti-rabbit secondary Alexa $546 \mathrm{Ab}$. Fluorescence images are shown in black and white. The analysed parasites are shown in the phase contrast image on the left (phase) while the merged images of the $L d$ Ran (red) and NUP (green) staining are shown on the right. A typical ROI used for quantitation of NUP and $L d$ Ran colocalisation is shown on the upper right corner of the green channel. A 1.5 fold magnification of the nucleus is shown in the insets of each image. 
661 B. $L d$ Ran localisation with respect to histones LeishH1 and LeishH3. 1. Wild-type $L$.

662

663

664

665

666

667

668

669

670

671

672

673

674

675

676

677

678

679

680

681

682

683

684

685

686

687

688

689

690

691

692

693

694

695

696

697

698

699

700

701

702

703

704

705

706

707

708

709 donovani promastigotes were stained for LeishH3 (LeishH3) with mouse specific LeishH3 $\mathrm{pAb}$ and an anti-mouse Alexa $488 \mathrm{Ab}$, and for $L d \mathrm{Ran}(L d \mathrm{Ran})$ with a rabbit anti- $L d \mathrm{Ran}$ pAb and an anti-rabbit Alexa $546 \mathrm{Ab}$. The average Pearson's correlation coefficient for the $L d$ Ran and LeishH3 intranuclear localisation was equal to $0.65(\mathrm{r}=0.65)$ and was calculated from 15 cells from 3 independent experiments. The average red in green co-localisation ( $L d$ Ran in LeishH3) was equal to $60 \%$, whereas the green in red (LeishH3 in $L d$ Ran) was $40 \%$. Typical ROIs are shown on the upper right corner of the green channel. 2. Wild-type L. donovani promastigotes were stained for $L d$ Ran ( $L d$ Ran), with an anti- $L d$ Ran specific mouse pAb and an anti-mouse Alexa 546 secondary Ab, and for LeishH1 (LeishH1) with the anti-LeishH1 specific rabbit pAb and an anti-rabbit secondary Alexa $488 \mathrm{Ab}$. The merged images of the LeishH1 (green) and $L d$ Ran (red) staining are shown on the right. The average Pearson's correlation coefficient for the $L d$ Ran and LeishH1 intranuclear localisation was equal to 0.9 $(\mathrm{r}=0.9)$ and was calculated from 15 cells from 3 independent experiments. The average red in green co-localisation ( $L d \mathrm{Ran}$ in LeishH1) was equal to $90 \%$, whereas the green in red (LeishH1 in $L d$ Ran) was $80 \%$. Typical ROIs are shown on the upper right corner of the red channel. 3. Wild-type L. donovani promastigotes were stained for nucleoporins (NUP), with an anti-nucleoporin specific $\mathrm{mAb}$ and an anti-mouse Alexa 546 secondary $\mathrm{Ab}$, and for LeishH1 (LeishH1) with the anti-LeishH1 specific rabbit $\mathrm{pAb}$ and an anti-rabbit secondary Alexa $488 \mathrm{Ab}$. The merged images of the LeishH1 (green) and NUP (red) staining are shown on the right. The analyzed parasites for all panels are shown in the phase contrast images on the left (phase).

\section{Fig. 4}

\section{LdRan interacts with LeishH1 in vitro}

A. Immunoblot analysis of proteins eluted from $L d$ Ran immobilised on Ni-NTA beads using anti-LeishH1, anti- LeishH2B and anti- LeishH3 Abs. Recombinant $L d$ Ran (Ni-NTA- $L d$ Ran) immobilised Ni-NTA beads, was incubated with a leishmanial protein extract. An equal volume of Ni-NTA beads (Ni-NTA) was incubated with an equal amount of leishmanial protein extract. Beads were subsequently washed and proteins eluted with imidazole. An amount of $10 \%$ from the protein lysate used per reaction was also used as positive control (10\% protein extract input). A1: Ponceau-S staining of the western blot showing the amounts of $L d$ Ran used per reaction. A2: Immunoblot analysis using anti-LeishH1 (LeishH1), antiLeishH2B (LeishH2B) and anti-LeishH3 (LeishH3) specific Abs to detect the presence of the corresponding histones. B. GST-Rabla, GST and $L d$ Ran $(2 \mu \mathrm{g}$ each) were immobilised on glutathione sepharose and Ni-NTA beads and incubated with leishmanial protein extract (2mg). An equal volume of Ni-NTA beads (Ni-NTA resin) incubated with leishmanial protein extract was loaded to check for non-specific protein precipitation. B1: Ponceau-S staining of the western blot showing the amounts of GST and GST-Rabla and $L d$ Ran, used per reaction. B2: Immunoblot analysis using an anti-LeishH1 specific Ab to detect the presence of LeishH1. 10\% from the protein lysate was used as a positive control to detect the presence of LeishH1. C. GST-LeishH1 $(2 \mu \mathrm{g})$ was immobilised on glutathione sepharose 4B beads and incubated with leishmanial protein extract (GST-LeishH1 + Lysate). GST protein $(2 \mu \mathrm{g})$ was immobilised on glutathione sepharose beads and incubated with an equal amount of protein extract (GST + Lysate). GST-LeishH1 and GST, were also loaded (GST-LeishH1 and GST) as negative controls. An amount of $5 \%$ from the protein lysate used per reaction was also used as positive control. C1: Ponceau-S staining of the Western Blot showing the amounts of GST and GST-LeishH1 used per reaction; C2: Immunoblot analysis using an 
710 anti- $L d$ Ran specific $A b$ to detect the presence of $L d$ Ran. The experiment was performed twice.

712

713

714 


\section{Fig.1}

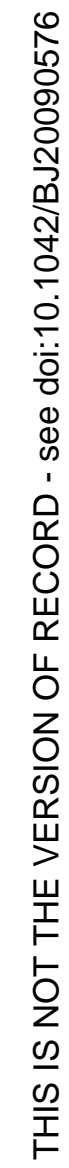

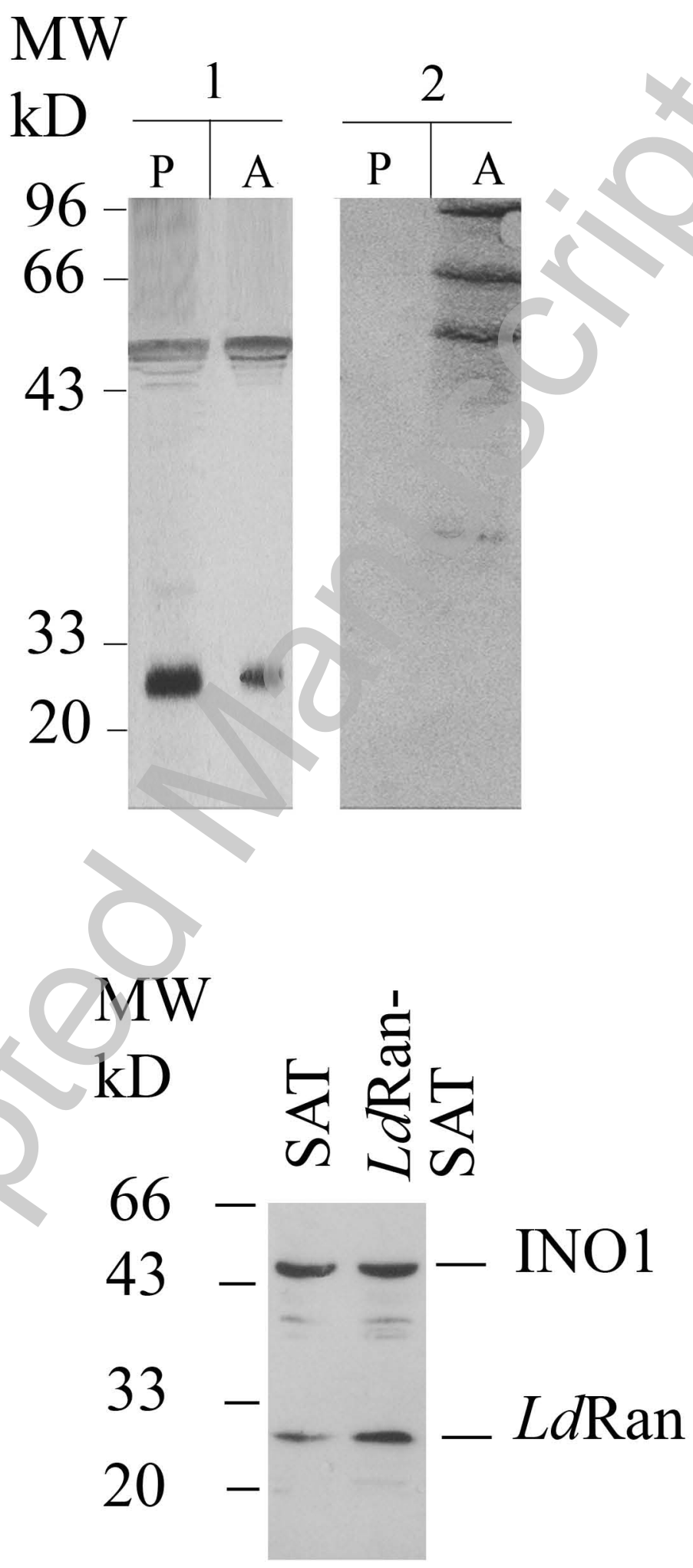


Fig. 2

Asynchronous

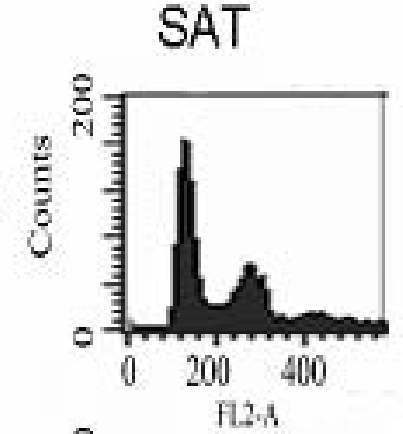

HU synchronised

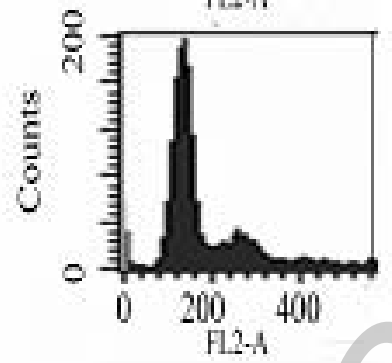

$4 \mathrm{~h}$

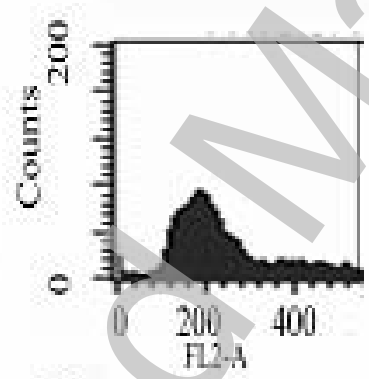

$6 \mathrm{~h}$

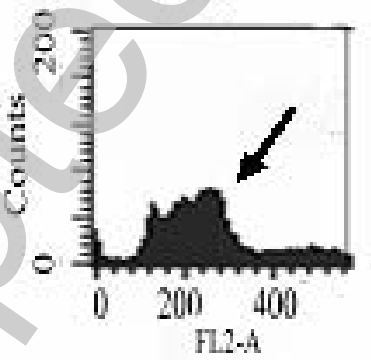

$10 \mathrm{~h}$

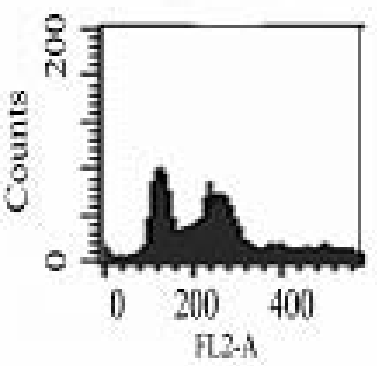

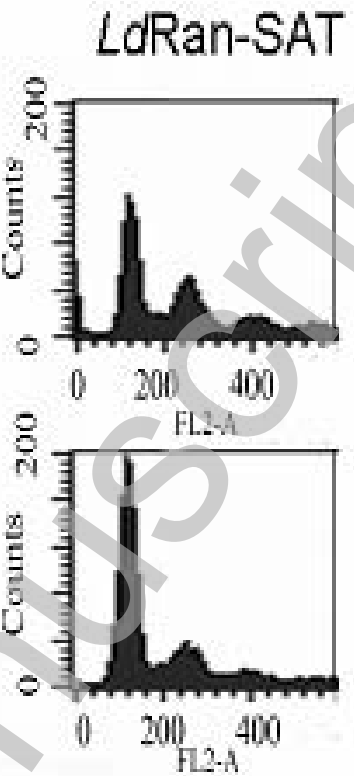

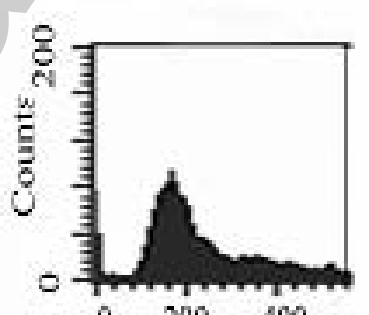

$\begin{array}{lll}0 & 200 & 400\end{array}$
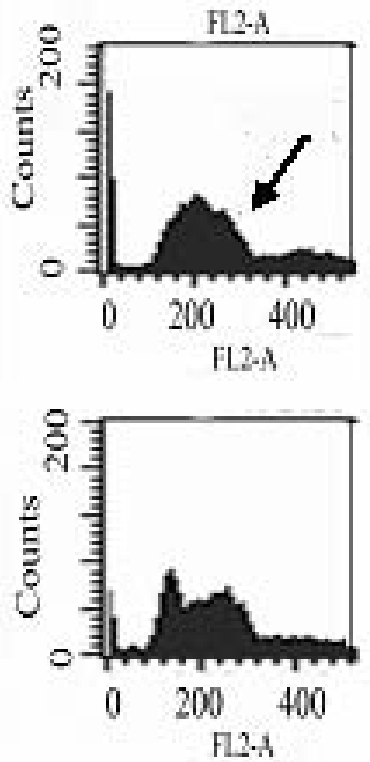
B Biochemical Journal Immediate Publication. Published on 22 Sep 2009 as manuscript BJ20090576

Fig. 3

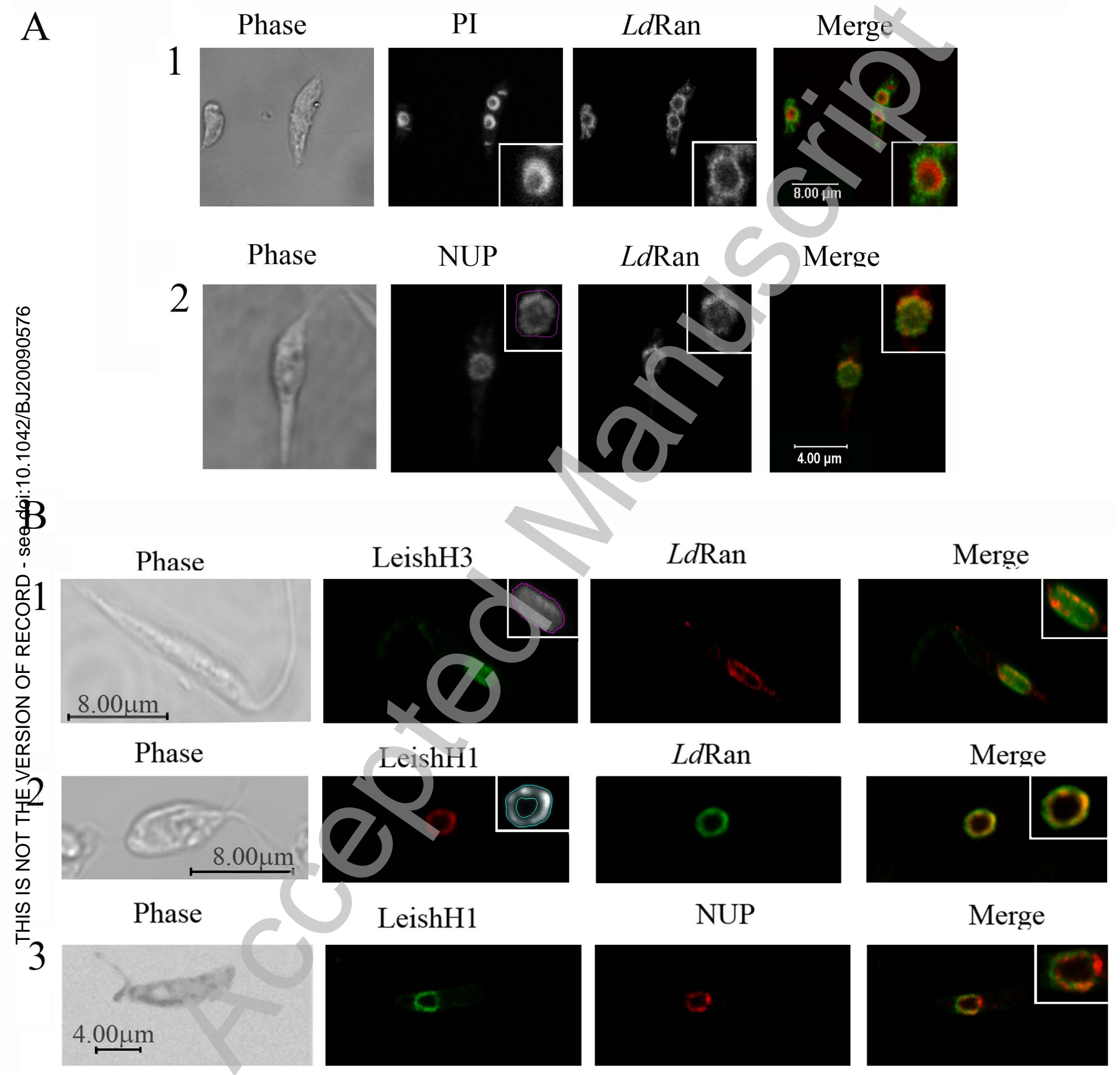

Licenced copy. Copying is not permitted, except with prior permission and as allowed by law. (C) 2009 The Authors Journal compilation (c) 2009 Portland Press Limited 
B) Biochemical Journal Immediate Publication. Published on 22 Sep 2009 as manuscript BJ20090576

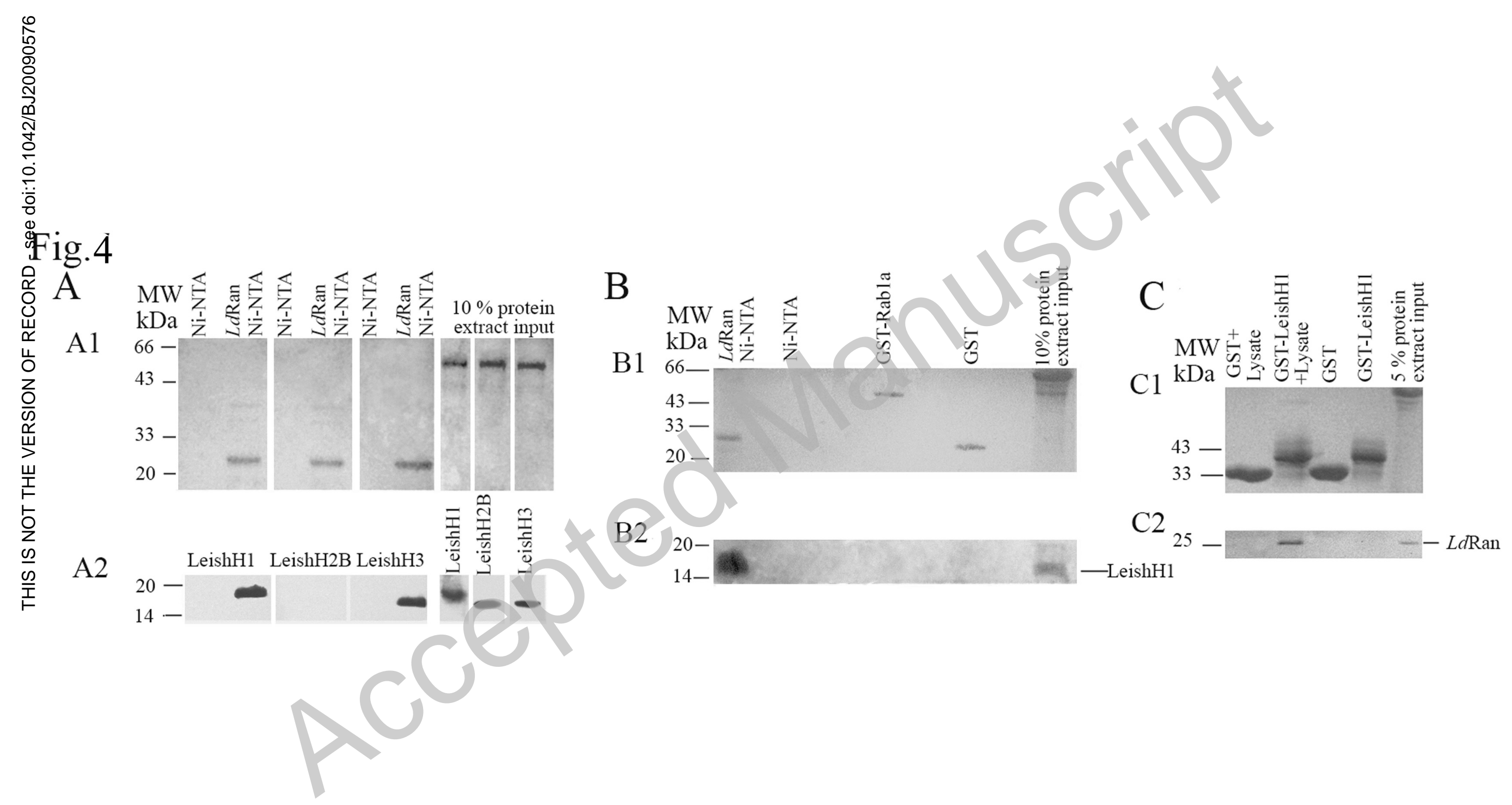

